\title{
Effect of two prophylaxis methods on adherence of Streptococcus mutans to microfilled composite resin and giomer surfaces
}

\author{
Soodabeh Kimyai ${ }^{1}$, Farzaneh Lotfipour ${ }^{2}$, Reza Pourabbas ${ }^{3}$, Alireza Sadr ${ }^{4}$, Saeedeh Nikazar ${ }^{5}$, Morteza Milani ${ }^{6}$
}

${ }^{1}$ DDS, MS: Associate Professor. Department of Operative Dentistry, School of Dentistry, Tabriz University of Medical Sciences, Tabriz, Iran

${ }^{2}$ Pharm.D, PhD: Assistant Professor. Department of Pharmaceutics, School of Pharmacy, Tabriz University of Medical Sciences, Tabriz, Iran

${ }^{3}$ DDS, MS: Associate Professor. Dental and Periodontal Research Center, Tabriz University of Medical Sciences, Tabriz, Iran ${ }^{4}$ DDS, PhD: Assistant Professor and Principal Investigator. Department of Cariology and Operative Dentistry, Global Center of Excellence, IRCMSTBD, Tokyo Medical and Dental University, Tokyo, Japan

${ }^{5}$ DDS Candidate. Department of Operative Dentistry, School of Dentistry, Tabriz University of Medical Sciences, Tabriz, Iran

${ }^{6} \mathrm{MSc}, \mathrm{PhD}$ Candidate. Department of Microbiology, School of Pharmacy, Tabriz University of Medical Sciences, Tabriz, Iran

Correspondence:

Department of Operative Dentistry,

Tabriz University of Medical Sciences,

School of Dentistry, Gholghasht Street,

Zip: 5166614713, Tabriz, Iran

soodabehkimyai@yahoo.com

Received: 16/03/2010

Accepted: $31 / 05 / 2010$

\author{
Kimyai S, Lotfipour F, Pourabbas R, Sadr A, Nikazar S, Milani M. Ef- \\ fect of two prophylaxis methods on adherence of Streptococcus mutans \\ to microfilled composite resin and giomer surfaces. Med Oral Patol Oral \\ Cir Bucal. 2011 Jul 1;16 (4):e561-7. \\ http://www.medicinaoral.com/medoralfree01/v16i4/medoralv16i4p561.pdf

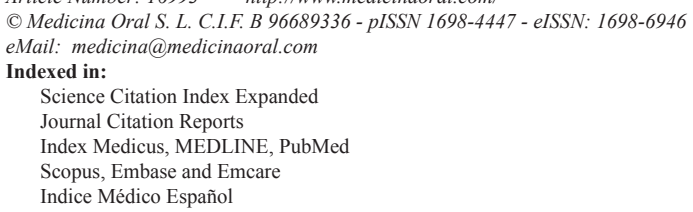

\begin{abstract}
Objectives: Surface attributes of a restoration play an important role in adherence of plaque bacteria. Prophylaxis methods may be involved in modification of or damaging the restoration surface. The aim of the present study was to evaluate the effect of two prophylaxis methods on adherence of Streptococcus mutans to the surface of two restorative materials. Study design: A total of 60 specimens were prepared from each material; a microfilled composite resin (HelioProgress) and a giomer (Beautifil II). For each material, the specimens were randomly divided into three groups $(\mathrm{n}=20)$. Group 1: no prophylaxis treatment (control); Group 2: prophylaxis with pumice and rubber cup; Group 3: prophylaxis with air-powder polishing device (APD). The surfaces of selected specimens from each group were evaluated under a scanning electron microscope (SEM), and the surface topography formed by the two prophylaxis methods was determined by atomic force microscopy (AFM). Adherence of Streptococcus mutans to the surface of specimens was determined by the plate counting method following immersion in a bacterial innoculum for 4 hours, rinsing and sonication. Data were analyzed by two-way ANOVA and post hoc Tukey test for multiple comparisons. Statistical significance was set at $\mathrm{P}<0.05$. Results: Bacterial adherence was significantly affected by both factors: restorative material type and prophylaxis method $(\mathrm{P}<0.0005)$. Mean bacterial adhesion was significantly higher in composite groups compared to corresponding giomer groups. Within each material, bacterial adherence was significantly lower in the control group compared to prophylaxis groups. Prophylaxis with pumice and rubber cup resulted in a significantly lower bacterial adherence compared to prophylaxis with APD. Conclusions: Based on the results of the present study, giomer specimens demonstrated lower bacterial adherence compared to composite resin specimens. In both materials, the highest bacterial adherence was observed with prophylaxis with APD, pumice and rubber cup and the control group, respectively.
\end{abstract}

Key words: Bacterial adherence, composite resin, giomer, prophylaxis methods. 


\section{Introduction}

Streptococcus mutans (S. mutans) is one of the most important cariogenic bacteria in the dental plaque. Adherence of the bacteria to the surface of dental restorations is a contributing factor to recurrent caries $(1,2)$. The surface topography of a restoration plays an important role in the adherence of bacteria present in the dental plaque (3-5); it has been reported that an increase in the surface roughness of restorations beyond a threshold $(0.2$ $\mu \mathrm{m})$ results in increased plaque retention, caries risk and gingival inflammation (6). Prophylaxis methods are aimed at mechanical removal of stains and plaque from tooth surfaces, especially in the vicinity of gingival tissues (7). These methods are factors involved in damaging and even destroying the surface of cervical restorations (8).

Use of pumice and rubber cup is the most common method to remove plaque and stains (7). Recently, the use of air-powder polishing device (APD) has gained popularity among dentists. APD is more effective in removal of plaque and stains, and a shorter time is needed compared to pumice and rubber cup technique (7). However, previous studies on various types of composite resins and glass-ionomer have reported that the APD produces a rougher surface compared to the pumice and rubber cup technique $(3,8)$.

Microfilled composite resins are among the choice restorative materials for cervical cavities, because of their low modulus of elasticity and low stress induction (9). Recently, a new generation of resin materials, namely "giomers", has been introduced for cervical restorations. These light-cured materials incorporate glass-ionomer fillers into the resin matrix. Giomers bear the advantages of both composite resins and glass-ionomers; they have excellent esthetics, good polishability, and biocompatibility and also render glass-ionomer properties, including fluoride release and fluoride recharge potential (10). Proper seal against bacterial microleakage and minimal mechanical and chemical irritation of the pulp are other advantages of giomers (11).

To date, few studies have evaluated the effect of prophylaxis methods on the adherence of $S$. mutans to the surface of restorations; therefore the aim of this study was to evaluate the effect of two prophylaxis methods on the adherence of $S$. mutans to the surface of microfilled composite resin and giomer. Two null hypotheses were tested: (1) Different prophylaxis methods do not affect the adherence of $S$. mutans to the surface of microfilled composite resin and giomer; and (2) $S$. mutans adheres to the surfaces of giomer and the microfilled composite resin in a similar manner.

\section{Materials and Methods}

The restorative materials used in this in vitro study were a microfilled composite resin (shade A3, HelioProgress;
Ivoclar Vivadent, Schaan, Liechtenstein) and a giomer (shade A3, Beautifil II; Shofu Dental Corporation, Osa$\mathrm{ka}$, Japan). A total of 60 specimens were prepared from each material. The specimens were cylindrical in shape with a diameter of $6 \mathrm{~mm}$ and a height of $2 \mathrm{~mm}$.

-Preparation of the specimens

A Teflon mold, with an inner diameter of $6 \mathrm{~mm}$ and a height of $2 \mathrm{~mm}$, was placed on a glass slab. Then, the restorative material was packed into the Teflon mold. A transparent matrix strip (Hawe Neos Dental, Bioggio, Switzerland) was placed on the surface of the restorative material to produce a smooth and uniform surface in all the specimens; a glass slide was then pressed on the Teflon mold containing the restorative material. Subsequently, the specimens were light-cured using Astralis 7 light-curing unit (Ivoclar Vivadent, FL-9494 Schaan, Liechtenstein) at a light intensity of $400 \mathrm{~mW} /$ $\mathrm{cm}^{2}$, with the light tip held perpendicular to the surface of the specimens for 40 seconds. The specimens were removed from the molds and were again light-cured from four directions (top, bottom and two sides) for 20 seconds each, to ensure proper polymerization.

\section{-Prophylaxis treatments}

After preparing 60 specimens from each material, they were randomly divided into three groups $(\mathrm{n}=20)$; composite specimens were distributed in groups $\mathrm{A}, \mathrm{B}$ and $\mathrm{C}$ and giomer specimens in groups D, E and F.

In the first groups of each material (groups A and D), no prophylaxis method was used.

In the second groups (groups B and E), the specimens underwent a prophylaxis procedure with pumice slurry containing a mixture of pumice powder (Kemdent, Swindon, Wiltshire, UK) and water; and a rubber cup (Stoddard, Letchworth, Hertfordshire, UK) for $12 \mathrm{sec}-$ onds (8) using a slow-speed (2000 rpm) handpiece. A new rubber cup was used for each specimen and the pumice slurry was replaced every 6 seconds (8). Subsequent to the prophylaxis procedure, the specimens were rinsed and placed in an ultrasonic bath for 10 minutes to clean the surfaces (12).

In the third groups (groups $\mathrm{C}$ and $\mathrm{F}$ ), the specimens underwent a prophylaxis procedure with APD (Air-Flow, Electronic Medical Systems, Nyon, Switzerland) for 12 seconds (8) at a distance of $10 \mathrm{~mm}$ from the surface and perpendicular to the surface, with regular powder in the first 6 seconds and with fine powder in the following 6 seconds. Subsequent to the prophylaxis procedure, the specimens were rinsed and placed in an ultrasonic bath for 10 minutes to clean the surfaces (12).

-Surface topography characterization and surface roughness

The specimens in the experimental groups underwent a surface topography characterization procedure under an atomic force microscope (AFM) (NanoScope II, Digital Instruments, Santa Barbara, CA, USA). Two 
additional specimens from each group were prepared for AFM characterization procedure. A silica nitride tip (with nominal radius of $50 \mathrm{~nm}$ and an apex angel of $45^{\circ}$ ) connected to a fixed substrate on a cantilever, was used for the AFM procedure. The images were recorded at a resolution of $256 \times 256$ pixels with a scan rate of $1.9 \mathrm{~Hz}$. Scans were carried out at surface quadrants of each specimen, consisting of areas of $10 \mu \mathrm{m} \times 10 \mu \mathrm{m}$. Three-dimensional data were analyzed with a dataanalysis software (NanoScope III, Version 5.12r2, Digital Instruments, Santa Barbara, CA, USA). The surface roughness for each group was reported in $\mathrm{nm}$ by the root mean square (rms) of the values of the surface departures of the sampling area.

\section{-Scanning electron microscope (SEM) observations}

Two additional specimens from the six experimental groups were prepared for surface evaluation under a scanning electron microscope. The specimens were gold-splutter coated and viewed under a SEM (Tescan, Vega II XMU, Brno, Czech Republic). In addition, the atomic composition of the uppermost surface of giomer and composite resin specimens in the experimental groups was determined using an energy dispersive $\mathrm{X}$ ray analyzer (Rontec $\mathrm{GmbH}$, Berlin, Germany) connected to the SEM.

All the specimens were prepared by one operator. In order to control microbial contamination, all the specimens were placed in microbial lamina under UV before bacterial adherence assay.

\section{-Bacterial adherence assay}

The standard S. mutans strains (PTCC 1683) used in the present study were purchased in lyophilized form from a commercial source. They were activated by culturing in sterile trypticase soy broth (Liofilchem s.r.l., Roseto degli Abruzzi, Teramo, Italy) supplemented with yeast extract (Difco Laboratories, Detroit, MI, USA); and then incubated for 48 hours at $37^{\circ} \mathrm{C}$. Single colonies from the plate were transferred into $4 \mathrm{~mL}$ of fluid trypticase soy broth supplemented with yeast external and incubated overnight at $37^{\circ} \mathrm{C}$. A centrifugation procedure at 3000 rpm was used for 15 minutes to harvest the cells. Then the cells were rinsed twice and re-suspended in Ringer solution to reach an optical density of approximately 0.3 at $540 \mathrm{~nm}$ with a spectrophotometer (Coleman Junior II, Coleman Instruments. Inc., Maywood, IL, USA) or bacterial concentrations of approximately $10^{8} \mathrm{CFU} / \mathrm{mL}$. The prepared innoculum was used for the in vitro adherence test according to the method used by Montanaro et al (13) with some modifications: The specimens were immersed, under aseptic conditions, in the bacterial suspension for 4 hours. Then the samples were retrieved from the suspension and rinsed three times with $5 \mathrm{~mL}$ of $0.9 \%$ normal saline to eliminate un-attached bacteria. Each sample was placed in a $15-\mathrm{mL}$ tube containing $1 \mathrm{~mL}$ of normal saline. The tubes were transferred to an ultrasonic bath cleaner (ModStar Sonic 1835, Italy) operating at $34 \mathrm{kHz}$ and $180 \mathrm{~W}$, and sonicated for 6 minutes to detach bacteria adhering to the surfaces of the specimens and to release them into the suspension. Finally the specimens were removed from the tubes and $100 \mu \mathrm{L}$ of the suspension containing detached bacteria was transferred onto a sterile plate for bacterial count via a standard pour plate technique. All the experiments were performed in triplicates.

-Statistical analysis

Two-way ANOVA was used for the statistical analysis of bacterial adherence and surface roughness data. A post hoc Tukey test was used for multiple comparisons. Statistical significance was set at $\mathrm{P}<0.05$.

\section{Results}

\section{-Bacterial adherence}

The results of adherence of $S$. mutans to composite resin and giomer surfaces are presented in Table 1. Significant differences were observed in bacterial adherence based on the type of material $\left(\mathrm{F}_{1.114}=113.53, \mathrm{P}<0.0005\right)$ and prophylaxis method $\left(\mathrm{F}_{2,114}=483.59, \mathrm{P}<0.0005\right)$. The interaction between these two factors was also statistically significant $\left(\mathrm{F}_{2,114}=10.98, \mathrm{P}<0.0005\right)$. Tukey test demonstrated statistically significant differences between groups $(\mathrm{P}<0.0005)$. In the giomer groups bacterial adherence was significantly lower than the corresponding composite resin groups. In the giomer and composite resin groups the highest bacterial adherence was observed with prophylaxis with APD, pumice and rubber cup and the control group, respectively.

-Surface topography characterization and surface roughness

Selected AFM images of the specimens from the six tested groups are shown in Figure 1 (A-F). The control group specimens revealed smooth surfaces in AFM images (Figs. 1, A, D), while specimens from other groups showed rough surfaces with macro- and micro-irregularities. Surface roughness values (mean \pm SD) for all the groups are presented in Table 1. The results of surface roughness test did not reveal any significant differences in relation to the type of the material $\left(\mathrm{F}_{1,42}=1.92\right.$, $\mathrm{P}=0.173$ ); however, there were significant differences in surface roughness in relation to the prophylaxis method used, regardless of the type of the material $\left(\mathrm{F}_{2}\right.$, ${ }_{42}=2763.58, \mathrm{P}<0.0005$ ). Multiple comparisons by Tukey test among subgroups within each material revealed significant differences between the prophylaxis methods employed $(\mathrm{P}<0.0005)$. There was no statistically significant interaction between the two factors involved (type of the material and type of the prophylaxis methods) $\left(\mathrm{F}_{2,42}=0.314, \mathrm{P}=0.732\right)$.

-SEM observation and surface composition

SEM images of the specimens in the tested groups are shown in Figure $2(\times 1000)$. Table 2 presents the atomic 


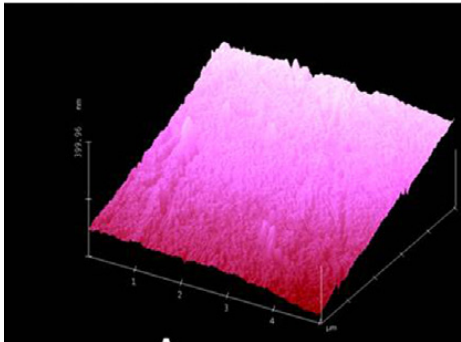

A

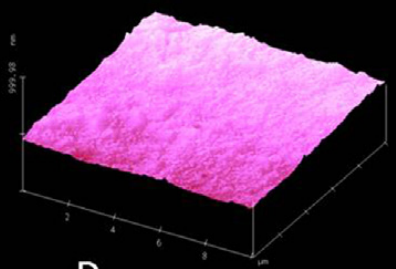

D

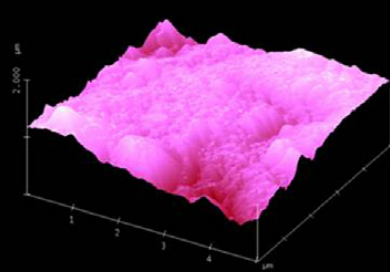

B

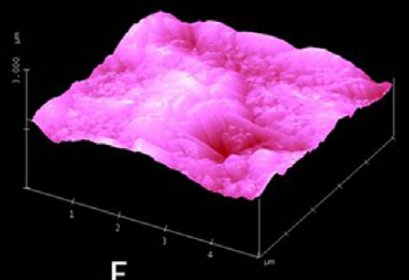

E

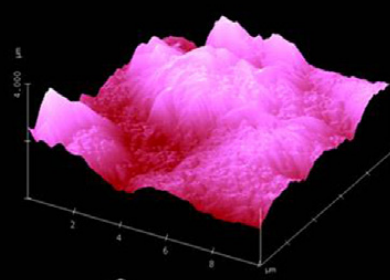

C

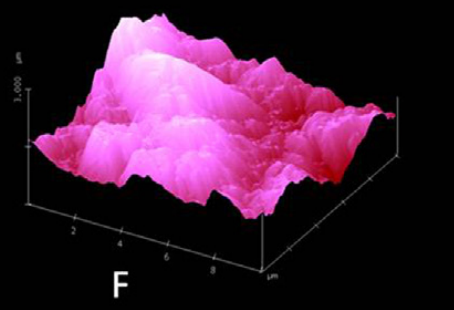

Fig. 1. AFM 3D topographical images of the surfaces of composite resin and giomer in the tested groups: A (composite, control), B (composite, pumice with rubber cup), C (composite, APD), D (giomer, control), E (giomer, pumice with rubber cup) and F (giomer, APD).
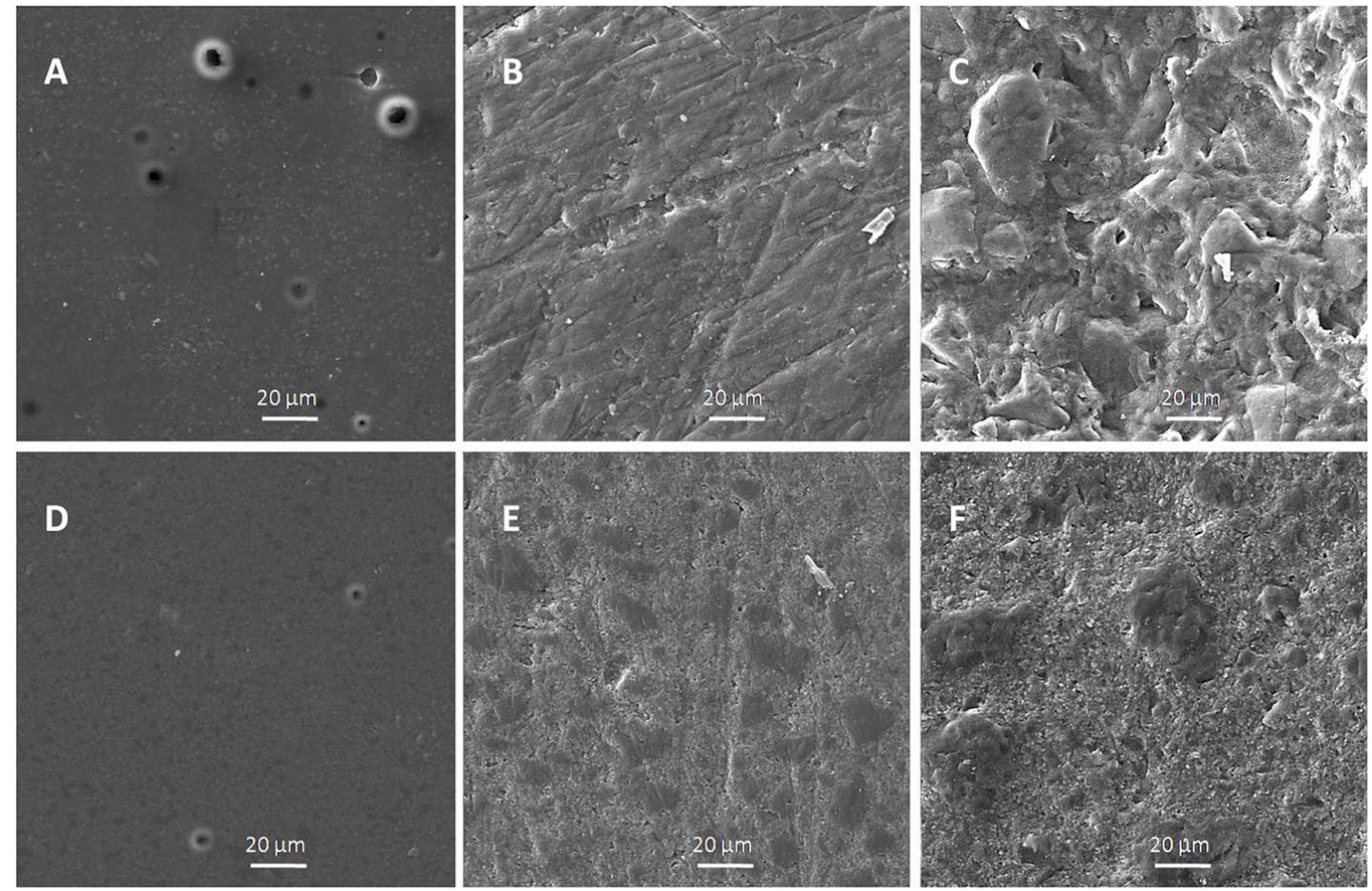

Fig. 2. SEM images of the surfaces of the un-treated composite specimen (A); composite specimen with pumice with rubber cup (B); composite specimen with APD (C); un-treated giomer specimen (D); giomer specimen with pumice with rubber cup (E); and giomer specimen with APD (F). 
composition of giomer and composite resin surfaces in the tested groups; the giomer specimens revealed higher amount of fluoride in the surface layer compared to composite resin specimens.

\section{Discussion}

Adherence of $S$. mutans to restoration surfaces results in bacterial plaque formation and recurrent caries (4). The results of the present study showed that in both giomers and composite resins, $S$. mutans adherence in the groups undergoing prophylaxis methods was higher than that in the controls. It was also revealed that the application of APD significantly increased bacterial adherence compared to pumice and rubber cup (Table 1). This finding might be attributed to the increased surface roughness of the tested materials. In the same context, the results of AFM analysis in this study confirmed those of the previous studies conducted on glass-ionomers and composite resins $(3,8)$; the surface roughness of both giomer and composite resin was significantly higher subsequent to prophylaxis with APD compared to prophylaxis with pumice/rubber cup. Moreover, surface roughness was higher subsequent to prophylaxis with pumice/rubber cup compared to the control group (Table 1). This finding might be attributed to the high pressure of air and water in APD, which results in the disintegration of filler-resin interface and debonding of fillers from the surface. It has also been reported that powder particles in APD can wear out the filler phase of composite resins (8).

Surface roughness of restorative materials has been reported as a factor contributing to bacterial adherence in several studies $(4,5,14-17)$. In a study carried out by Ikeda et al, it was reported that smooth resin composite surfaces exhibit lower bacterial adherence and accumulation in comparison to rougher resin composite surfaces (17). Moreover, Ono et al demonstrated that smooth resin composite surfaces have an important role in retarding the biofilm adherence and growth (15).

Contrary to the results of the present study, in a study carried out by Meier et al surface roughness of various ceramics did not influence bacterial adherence (4). This discrepancy might be attributed to the fact that in that study the initial surface roughness of various ceramics had been quantified and no roughening procedures had been carried out, resulting in a smaller range of roughness values investigated. However, in the present study the surface of the specimens underwent prophylaxis procedures. Bacterial adherence is probably under the influence of increased surface roughness because greater surface area and protective sites are provided for bacterial colonization (15).

In addition, physico-chemical properties and free surface energy of the substrate can influence bacterial adherence $(4,5)$. Meanwhile, the effect of surface roughness on bacterial adherence has been reported to be greater than that of free surface energy (4). In the present study, surface analysis under SEM revealed that prophylaxis of the specimens resulted in alterations in surface elements and increased the ratio of minerals (Al $+\mathrm{Si}$ ) (which have a positive charge) on the surface (Table 2). It has been reported that an increase in the polarity of substrate surface can increase free surface energy leading to an increase in bacterial adherence (5).

The results of the present study showed that bacterial adherence in all the giomer groups was significantly lower than that in the corresponding composite resin groups. According to the results of AFM analysis both giomer and composite resin demonstrated similar wear resistance values in prophylaxis procedures, and there were no significant differences in surface roughness between corresponding giomer and composite resin specimens (Table 1 and Fig. 1). Differences in bacterial adherence might also be attributed to different chemical compositions of the two materials. Previous studies have demonstrated the effect of chemical composition of materials on bacterial adherence $(4,5)$. The composite resin used in the present study (HelioProgress) is devoid of fluoride according to the manufacturer, whereas giomer (Beautifil II) is a fluoride-releasing material which contains PRG (pre-reacted glass) fillers with surface reaction (S-PRG) (10). It has even been reported that this giomer releases more fluoride compared to giomers containing PRG fillers with full reaction (F-PRG) (14). In the same context, surface analysis by SEM showed that giomer specimens have a much higher content of fluoride compared to composite resin specimens (Table 2). Antibacterial effects of fluoride have already been reported (18). It has been demonstrated that the water insoluble glucan in $S$. mutans biofilm is affected by the fluoride released from giomer (15). In addition, Palenik et al have reported the inhibitory effect of glass-ionomer on bacterial growth and adherence as a result of fluoride release from this material (19). Giomer contains fluoridated glass fillers with glass-ionomer matrix. This matrix has a high content of fluoride complex, and water easily penetrates into it, which results in the release of large quantities of fluoride (20). Previous studies have reported greater fluoride release from giomers in comparison to composite resins $(20,21)$.

Based on the results of this study, re-polishing of giomer and composite resin restorations subsequent to the prophylaxis treatments investigated might be necessary to resist bacterial adherence in clinical settings. Meanwhile, it seems that maintaining a well-polished surface to reduce the bacterial adherence is more crucial for composite resin restorations compared to giomer restorations, due to fluoride release property in giomer restorative materials. It is proposed that the released fluoride might postpone the initial bacterial adherence and further plaque formation. 
Table 1. Number of bacteria adhering to the surface of composite resin and giomer in the tested groups (mean \pm standard deviations) and surface roughness obtained from the AFM images for the tested groups.

\begin{tabular}{|c|c|c|c|c|}
\hline \multirow{2}{*}{$\begin{array}{c}\text { Prophylaxis } \\
\text { treatment }\end{array}$} & \multicolumn{2}{|c|}{ Number of bacteria (Mean \pm} & \multicolumn{2}{c|}{ Surface roughness (Mean \pm SD) } \\
\cline { 2 - 5 } & \multicolumn{2}{|c|}{ Material } & Material \\
\cline { 2 - 5 } & Composite & Giomer & Composite & $17.37 \pm 1.38^{\mathrm{a}}$ \\
\hline \multirow{2}{*}{ None } & $\begin{array}{c}209.20 \pm \\
47.25^{*}\end{array}$ & $\begin{array}{c}128.80 \pm \\
32.45^{*}\end{array}$ & $18.66 \pm 1.12^{\mathrm{a}}$ & \\
\hline Pumice with & $425.95 \pm$ & $264.45 \pm$ & $134.39 \pm 11.21^{\mathrm{b}}$ & $130.75 \pm 6.39^{\mathrm{b}}$ \\
rubber cup & $65.47^{*}$ & $41.93^{*}$ & & \\
\hline APD & $557.10 \pm$ & $498.05 \pm$ & $275.58 \pm 10.62^{\mathrm{c}}$ & $268.88 \pm 16.74^{\mathrm{c}}$ \\
& $46.80^{*}$ & $66.57^{*}$ & & \\
\hline
\end{tabular}

Asterisk (*) indicates significant differences in pairwise comparisons in bacterial adherence between all the groups $(\mathrm{P}<0.001)$. Mean values of surface roughness with dissimilar letters are statistically significant at $\mathrm{P}<0.05$.

Table 2. Surface composition (at \%) of composite resin and giomer in the tested groups.

\begin{tabular}{|l|c|c|c|c|c|c|c|c|c|}
\hline \multirow{2}{*}{ Groups } & \multicolumn{7}{|c|}{ Surface elements } \\
\cline { 2 - 10 } & $\mathbf{C}$ & $\mathbf{O}$ & $\mathbf{F}$ & $\mathbf{A l}$ & $\mathbf{S i}$ & $\mathbf{N a}$ & $\mathbf{K}$ & $\mathbf{C a}$ & Si +Al \\
\hline Group A & 65.81 & 30.59 & 0.50 & 0.01 & 3.09 & - & - & - & 3.10 \\
\hline Group B & 70.07 & 25.41 & 1.30 & 0.13 & 3.09 & - & - & - & 3.22 \\
\hline Group C & 61.45 & 33.49 & 0.00 & - & 5.06 & - & - & - & 5.06 \\
\hline Group D & 67.37 & 24.26 & 5.02 & 0.76 & 2.60 & - & - & - & 3.36 \\
\hline Group E & 57.34 & 21.23 & 8.58 & 3.43 & 7.65 & 1.05 & 0.37 & 0.34 & 11.08 \\
\hline Group F & 58.14 & 25.52 & 7.80 & 2.22 & 5.26 & 0.77 & 0.15 & 0.14 & 7.48 \\
& & & & & & & & & \\
\hline
\end{tabular}

The present study investigated bacterial adherence of giomer in the short term. While a high fluoride recharge capacity has been suggested for the giomer (20), the long-term release of fluoride from giomer is questionable (22). Moreover, in a previous study it has been demonstrated that $S$. mutans growth on aged composite resins increases surface roughness, further accelerating biofilm accumulation (23). Early bacterial adherence (first 48 hours) and prolonged biofilm formation might be affected differently by surfaces texture of restorative materials. Therefore, further long-term investigations using more clinically relevant biofilm formation mechanisims [such as artificial mouth system $(15,17)]$ are warranted to evaluate bacterial adherence to giomers.

\section{Conclusion}

Considering the limitations of the present study it can be concluded that:

Both prophylaxis methods (pumice and rubber cup vs. APD) resulted in an increase in bacterial adherence to 
giomer and composite resin in comparison to the control group.

Prophylaxis with APD resulted in greater bacterial adherence compared to prophylaxis with pumice and rubber cup.

Bacterial adherence in giomer groups was lower compared to corresponding composite resin groups regardless of the prophylaxis technique and the generated surface roughness.

\section{References}

1. López-Píriz R, Aguilar L, Giménez MJ. Management of odontogenic infection of pulpal and periodontal origin. Med Oral Patol Oral Cir Bucal. 2007;12:E154-9.

2. Yamamoto K, Ohashi S, Taki E, Hirata K. Adherence of oral streptococci to composite resin of varying surface roughness. Dent Mater J. 1996;15:201-4.

3. Wu SS, Yap AU, Chelvan S, Tan ES. Effect of prophylaxis regimens on surface roughness of glass ionomer cements. Oper Dent. 2005;30:180-4.

4. Meier R, Hauser-Gerspach I, Lüthy H, Meyer J. Adhesion of oral streptococci to all-ceramics dental restorative materials in vitro. $\mathrm{J}$ Mater Sci Mater Med. 2008;19:3249-53.

5. Carlén A, Nikdel K, Wennerberg A, Holmberg K, Olsson J. Surface characteristics and in vitro biofilm formation on glass ionomer and composite resin. Biomaterials. 2001;22:481-7.

6. Bollen CM, Lambrechts P, Quirynen M. Comparison of surface roughness of oral hard materials to the threshold surface roughness for bacterial plaque retention: a review of the literature. Dent Mater. 1997;13:258-69.

7. Agger MS, Hörsted-Bindslev P, Hovgaard O. Abrasiveness of an air-powder polishing system on root surfaces in vitro. Quintessence Int. 2001;32:407-11.

8. Yap AU, Wu SS, Chelvan S, Tan ES. Effect of hygiene maintenance procedures on surface roughness of composite restoratives. Oper Dent. 2005;30:99-104.

9. Calheiros FC, Sadek FT, Braga RR, Cardoso PE. Polymerization contraction stress of low-shrinkage composites and its correlation with microleakage in class V restorations. J Dent. 2004;32:407-12.

10. Deliperi S, Bardwell DN, Wegley C, Congiu MD. In vitro evaluation of giomers microleakage after exposure to $33 \%$ hydrogen peroxide: self-etch vs total-etch adhesives. Oper Dent. 2006;31:227-32. 11. Sunico MC, Shinkai K, Katoh Y. Two-year clinical performance of occlusal and cervical giomer restorations. Oper Dent. 2005;30:282-9.

12. Lai YL, Lin YC, Chang CS, Lee SY. Effects of sonic and ultrasonic scaling on the surface roughness of tooth-colored restorative materials for cervical lesions. Oper Dent. 2007;32:273-8.

13. Montanaro L, Campoccia D, Rizzi S, Donati ME, Breschi L, Prati C, et al. Evaluation of bacterial adhesion of Streptococcus mutans on dental restorative materials. Biomaterials. 2004;25:4457-63.

14. Daneshmehr L, Matin K, Nikaido T, Tagami J. Effects of root dentin surface coating with all-in-one adhesive materials on biofilm adherence. J Dent. 2008;36:33-41.

15. Ono M, Nikaido T, Ikeda M, Imai S, Hanada N, Tagami J, et al. Surface properties of resin composite materials relative to biofilm formation. Dent Mater J. 2007;26:613-22.

16. Teughels W, Van Assche N, Sliepen I, Quirynen M. Effect of material characteristics and/or surface topography on biofilm development. Clin Oral Implants Res. 2006;17 Suppl 2:68-81.

17. Ikeda M, Matin K, Nikaido T, Foxton RM, Tagami J. Effect of surface characteristics on adherence of $S$. mutans biofilms to indirect resin composites. Dent Mater J. 2007;26:915-23.

18. Yap AU, Mok BY. Effects of professionally applied topical fluorides on surface hardness of composite-based restoratives. Oper Dent. 2002;27:576-81.
19. Palenik CJ, Behnen MJ, Setcos JC, Miller CH. Inhibition of microbial adherence and growth by various glass ionomers in vitro. Dent Mater. 1992;8:16-20.

20. Itota T, Carrick TE, Yoshiyama M, McCabe JF. Fluoride release and recharge in giomer, compomer and resin composite. Dent Mater. 2004:20:789-95.

21. Gonzalez Ede H, Yap AU, Hsu SC. Demineralization inhibition of direct tooth-colored restorative materials. Oper Dent. 2004;29:57885 .

22. Yap AU, Tham SY, Zhu LY, Lee HK. Short-term fluoride release from various aesthetic restorative materials. Oper Dent. 2002;27:25965 .

23. Beyth N, Bahir R, Matalon S, Domb AJ, Weiss EI. Streptococcus mutans biofilm changes surface-topography of resin composites. Dent Mater. 2008;24:732-6.

\section{Acknowledgments}

The authors would like to extend their gratitude to the Research Vice Chancellor at Tabriz University of Medical Sciences for their financial support. Furthermore, the authors would like to thank Dr. M Abdolrahimi (DDS), who edited the English manuscript of this article and Dr. MR Naimi-Jamal for his assistance in AFM imaging. The authors also thank Shofu Dental Corporation for supplying the giomer material. 